\title{
Some analytical models of radiating collapsing spheres
}

\author{
L. Herrera ${ }^{1 *}$ A Di Prisco ${ }^{1 \dagger}$ and J. Ospino ${ }^{2 \ddagger}$ \\ ${ }^{1}$ Escuela de Física, Facultad de Ciencias, \\ Universidad Central de Venezuela, Caracas, Venezuela. \\ ${ }^{2}$ Area de Física Teórica. Facultad de Ciencias,Universidad de Salamanca \\ Salamanca, Spain.
}

June 24, 2021

\begin{abstract}
We present some analytical solutions to the Einstein equations, describing radiating collapsing spheres in the diffusion approximation. Solutions allow for modeling physical reasonable situations. The temperature is calculated for each solution, using a hyperbolic transport equation, which permits to exhibit the influence of relaxational effects on the dynamics of the system.
\end{abstract}

\footnotetext{
*e-mail: laherrera@movistar.net.ve

$\dagger$ e-mail: adiprisc@fisica.ciens.ucv.ve

‡e-mail: jhozcrae@usal.es
} 


\section{Introduction}

Our purpose in this work is to provide some analytical solutions to Einstein equations, describing collapsing dissipative spheres in the difussion approximation. Such solutions may serve as test-bed for numerical relativity, and for probing cosmic censorship and hoop conjecture, among other important issues, and represent a natural tool to bring out the influence of dissipation on the evolution of a collapsing object.

Analytical solutions although generally are found, either for too simplistic equations of state and/or under additional heuristic assumptions whose justification is usually uncertain, are more suitable for a general discussion than purely numerical solutions, which usually hinder to catch qualitative, aspects of the process.

Therefore it seems useful to consider analytical models which are relatively simple to analyze but still contain some of the essential features of a realistic situation.

Our endeavour is further justified by the following two considerations:

- It is already an established fact, that gravitational collapse is a highly dissipative process (see [1], [2], [3] and references therein). This dissipation is required to account for the very large (negative) binding energy of the resulting compact object (of the order of $-10^{53} \mathrm{erg}$ )

Indeed, it appears that the only plausible mechanism to carry away the bulk of the binding energy of the collapsing star, leading to a neutron star or black hole is neutrino emission [4].

- In the diffusion approximation, it is assumed that the energy flux of radiation (as that of thermal conduction) is proportional to the gradient of temperature. This assumption is in general very sensible, since the mean free path of particles responsible for the propagation of energy in stellar interiors is in general very small as compared with the typical length of the object. Thus, for a main sequence star as the sun, the mean free path of photons at the centre, is of the order of $2 \mathrm{~cm}$. Also, the mean free path of trapped neutrinos in compact cores of densities

about $10^{12}$ g. $\mathrm{cm}^{-3}$ becomes smaller than the size of the stellar core $[5,6]$.

Furthermore, the observational data collected from supernovae 1987A 
indicates that the regime of radiation transport prevailing during the emission process, is closer to the diffusion approximation than to the streaming out limit [7].

Accordingly we shall restrict here to this later case, being aware that there are situations in stellar evolution where that approximation fails.

During their evolution, self-gravitating objects may pass through phases of intense dynamical activity, with time scales of the order of magnitude of (or even smaller than) the hydrostatic time scale, and for which the quasistatic approximation is clearly not reliable, e.g., the collapse of very massive stars [8], and the quick collapse phase preceding neutron star formation, see for example [9] and references therein. In these cases it is mandatory to take into account terms which describe departure from equilibrium, i.e. a full dynamic description has to be used.

Here we are mainly concerned with the quick collapse phase, which implies that we have to appeal to a hyperbolic theory of dissipation. The use of a hyperbolic theory of dissipation is further justified by the necessity of overcoming the difficulties inherent to parabolic theories (see references [1], [10]-[23] and references therein). Doing so we shall be able to give a description of processes occuring before thermal relaxation.

Many analytical solutions of Einstein's field equations with dissipative fluids carrying heat flow have been studied (see [24] for references up to 1989 and [25], [26] for more recent ones).

In this vein here we present some models of conformally flat dissipative spherical collapse with shear-free motion. We match our models to a radiating null field described by the outgoing Vaidya spacetime.

Although the shear free and the conformally flat conditions are introduced here in order to simplify calculations, it is worth noticing that these conditions generalize physical assumptions widely used in astrophysics. Indeed, the shear free condition in the Newtonian regime describes the homologous evolution and the role of shear has been extensively considered in general relativity [27]. On the other hand it is well known that the conformally flat condition implies in the perfect fluid case the homogeneity of the energy density distribution [2].

The paper is organized as follows. In section 2 the field equations, conventions and junction conditions are presented; in section 3 we present the general solution corresponding to the conformal flatness condition; in sec- 
tions 4 and 5, particular analytical solutions are given, and a specific model is constructed from one of them; finally a brief conclusion is presented.

\section{The fluid distribution and the interior space- time}

We assume a sphere of collapsing perfect fluid with heat flow. Its spherical surface $\Sigma$ has center 0 and is filled with radially moving perfect fluid conducting heat flow, so having energy momentum tensor

$$
T_{\alpha \beta}=(\mu+p) w_{\alpha} w_{\beta}+p g_{\alpha \beta}+q_{\alpha} w_{\beta}+w_{\alpha} q_{\beta},
$$

where $\mu$ and $p$ are the proper density and pressure of the fluid, $w_{\alpha}$ its unit four-velocity, $q_{\alpha}$ the heat conduction satisfying $q_{\alpha} w^{\alpha}=0$ and $g_{\alpha \beta}$ is the metric tensor of spacetime.

We choose comoving coordinates within $\Sigma$ and impose shear-free fluid motion which allows the metric be written in the form (see [28] for details)

$$
d s^{2}=-A^{2} d t^{2}+B^{2}\left[d r^{2}+r^{2}\left(d \theta^{2}+\sin ^{2} \theta d \phi^{2}\right)\right],
$$

where $A$ and $B$ are only functions of $r$ and $t$. We number the coordinates $x^{0}=t, x^{1}=r, x^{2}=\theta$ and $x^{3}=\phi$ and then we have the four-velocity given by

$$
w_{\alpha}=-A \delta_{\alpha}^{0}
$$

and the heat flows radially,

$$
q^{\alpha}=q \delta_{1}^{\alpha}
$$

where $q$ is a function of $r$ and $t$. In these coordinates the equation of the boundary surface $\Sigma$ is given by $r=r_{\Sigma}=$ constant.

The spacetime described by (2) has the following non-null components of the Weyl tensor $C_{\alpha \beta \gamma \delta}$,

$$
C_{2323}=\frac{r^{4}}{3} B^{2} \sin ^{2} \theta\left[\left(\frac{A^{\prime}}{A}-\frac{B^{\prime}}{B}\right)\left(\frac{1}{r}+2 \frac{B^{\prime}}{B}\right)-\left(\frac{A^{\prime \prime}}{A}-\frac{B^{\prime \prime}}{B}\right)\right],
$$

and

$$
\begin{gathered}
C_{2323}=-r^{4}\left(\frac{B}{A}\right)^{2} \sin ^{2} \theta C_{0101}=2 r^{2}\left(\frac{B}{A}\right)^{2} \sin ^{2} \theta C_{0202} \\
=2 r^{2}\left(\frac{B}{A}\right)^{2} C_{0303}=-2 r^{2} \sin ^{2} \theta C_{1212}=-2 r^{2} C_{1313}
\end{gathered}
$$


where the primes stand for differentiation with respect to $r$ and dot stands for differentiation with respect to $t$.

\subsection{The field equations}

The non null components of Einstein's field equations $G_{\alpha \beta}=8 \pi T_{\alpha \beta}$, where $G_{\alpha \beta}$ is the Einstein tensor and $T_{\alpha \beta}$ is given by (1), with metric (2) are

$$
\begin{array}{r}
G_{00}=-\frac{A^{2}}{B^{2}}\left[2 \frac{B^{\prime \prime}}{B}-\left(\frac{B^{\prime}}{B}\right)^{2}+\frac{4}{r} \frac{B^{\prime}}{B}\right]+3\left(\frac{\dot{B}}{B}\right)^{2}=8 \pi \mu A^{2}, \\
G_{11}=\left(\frac{B^{\prime}}{B}\right)^{2}+\frac{2}{r} \frac{B^{\prime}}{B}+2 \frac{A^{\prime}}{A} \frac{B^{\prime}}{B}+\frac{2}{r} \frac{A^{\prime}}{A} \\
+\frac{B^{2}}{A^{2}}\left[-2 \frac{\ddot{B}}{B}-\left(\frac{\dot{B}}{B}\right)^{2}+2 \frac{\dot{A}}{A} \frac{\dot{B}}{B}\right]=8 \pi p B^{2}, \\
G_{22}=\frac{G_{33}}{\sin ^{2} \theta}=r^{2}\left[\frac{A^{\prime \prime}}{A}+\frac{1}{r} \frac{A^{\prime}}{A}+\frac{B^{\prime \prime}}{B}-\left(\frac{B^{\prime}}{B}\right)^{2}+\frac{1}{r} \frac{B^{\prime}}{B}\right] \\
+r^{2} \frac{B^{2}}{A^{2}}\left[-2 \frac{\ddot{B}}{B}-\left(\frac{\dot{B}}{B}\right)^{2}+2 \frac{\dot{A}}{A} \frac{\dot{B}}{B}\right]=8 \pi p r^{2} B^{2}, \\
G_{01}=-2\left(\frac{\dot{B}}{A B}\right)^{\prime} A=-8 \pi q A B^{2} .
\end{array}
$$

The mass function $m(r, t)$ of Cahill and McVittie [29] is obtained from the Riemann tensor component $R_{23}{ }^{23}$ and it is for metric (2)

$$
m(r, t)=\frac{(r B)^{3}}{2} R_{23}^{23}=\frac{r^{3} B}{2}\left[\left(\frac{\dot{B}}{A}\right)^{2}-\left(\frac{B^{\prime}}{B}\right)^{2}\right]-r^{2} B^{\prime} .
$$

For studying the dynamical properties of the field equations and following Misner and Sharp [30], let us introduce the velocity $U$ of the collapsing fluid as

$$
U=r D_{t} B(<0 \text { in the case of collapse })
$$

where the proper time derivative $D_{t}$, is given by

$$
D_{t}=\frac{1}{A} \frac{\partial}{\partial t} .
$$




\section{$2.2 \quad$ Junction conditions}

If the collapsing fluid lies within a spherical surface $\Sigma$ it must be matched to a suitable exterior. Since heat will be leaving the fluid across $\Sigma$, the exterior is not vacuum, but the outgoing Vaidya spacetime which models the radiation and has metric

$$
d s^{2}=-\left[1-\frac{2 m(v)}{\rho}\right] d v^{2}-2 d v d \rho+\rho^{2}\left(d \theta^{2}+\sin ^{2} \theta d \phi^{2}\right),
$$

where $m(v)$ is the total mass inside $\Sigma$ and is a function of the retarded time $v$. In (14) $\rho$ is a radial coordinate given in a non-comoving frame.

The conditions for the matching of these two spacetimes (2) and (14), are the Darmois conditions [31], which using the field equations (8-10) and the mass function (11) imply [24]

$$
\begin{array}{r}
p_{\Sigma}=(q B)_{\Sigma} \\
(q B)_{\Sigma}=\frac{1}{4 \pi}\left(\frac{L}{\rho^{2}}\right)_{\Sigma} \\
(r B)_{\Sigma}=\rho_{\Sigma} \\
\left(\frac{r^{3}}{2} \frac{B \dot{B}^{2}}{A^{2}}-\frac{r^{3}}{2} \frac{B^{\prime 2}}{B}-r^{2} B^{\prime}\right)_{\Sigma}=m(v), \\
A_{\Sigma} d t=\left(1-\frac{2 m}{\rho}+2 \frac{d \rho}{d v}\right)_{\Sigma}^{1 / 2} d v
\end{array}
$$

where $L$ is defined as the total luminosity of the collapsing sphere as measured on its surface and is given by

$$
L=L_{\infty}\left(1-\frac{2 m}{\rho}+2 \frac{d \rho}{d v}\right)^{-1}
$$

and where

$$
L_{\infty}=\frac{d m}{d v}
$$

is the total luminosity measured by an observer at rest at infinity. 


\section{Conformally flat solution}

Here we impose conformal flatness to the spacetime given by (2), i.e. all its Weyl tensor components must be zero valued. Then it can be shown that metric functions $A$ and $B$ take the form (see [28] for details)

$$
A=\left[C_{1}(t) r^{2}+1\right] B
$$

where $C_{1}$ is an arbitrary function of $t$ and

$$
B=\frac{1}{C_{2}(t) r^{2}+C_{3}(t)}
$$

where $C_{2}$ and $C_{3}$ are arbitrary functions of $t$.

Substituting solution (22) and (23) into (8), (9) and (11) we obtain,

$$
\begin{array}{r}
8 \pi \mu=3\left(\frac{\dot{C}_{2} r^{2}+\dot{C}_{3}}{C_{1} r^{2}+1}\right)^{2}+12 C_{2} C_{3}, \\
8 \pi p=\frac{1}{\left(C_{1} r^{2}+1\right)^{2}}\left[2\left(\ddot{C}_{2} r^{2}+\ddot{C}_{3}\right)\left(C_{2} r^{2}+C_{3}\right)-3\left(\dot{C}_{2} r^{2}+\dot{C}_{3}\right)^{2}\right. \\
\left.-2 \frac{\dot{C}_{1}}{C_{1} r^{2}+1}\left(\dot{C}_{2} r^{2}+\dot{C}_{3}\right)\left(C_{2} r^{2}+C_{3}\right) r^{2}\right] \\
+\frac{4}{C_{1} r^{2}+1}\left[C_{2}\left(C_{2}-2 C_{1} C_{3}\right) r^{2}+C_{3}\left(C_{1} C_{3}-2 C_{2}\right)\right] \\
8 \pi q=4\left(\dot{C}_{3} C_{1}-\dot{C}_{2}\right)\left(\frac{C_{2} r^{2}+C_{3}}{C_{1} r^{2}+1}\right)^{2} r
\end{array}
$$

Finally, from (15), (25) and (26) we have

$$
\begin{aligned}
\left\{\ddot{C}_{2} r^{2}\right. & +\ddot{C}_{3}-\frac{3}{2} \frac{\left(\dot{C}_{2} r^{2}+\dot{C}_{3}\right)^{2}}{C_{2} r^{2}+C_{3}}-\frac{\dot{C}_{1} r^{2}\left(\dot{C}_{2} r^{2}+\dot{C}_{3}\right)}{C_{1} r^{2}+1}-2\left(\dot{C}_{3} C_{1}-\dot{C}_{2}\right) r \\
& \left.+2 \frac{\left(C_{1} r^{2}+1\right)}{C_{2} r^{2}+C_{3}}\left[C_{2}\left(C_{2}-2 C_{1} C_{3}\right) r^{2}+C_{3}\left(C_{1} C_{3}-2 C_{2}\right)\right]\right\}_{\Sigma}=0
\end{aligned}
$$

Please note that a misprint in (27) appearing in [28] has been corrected here.

In the following sections we shall obtain some analytical solutions satisfying (15)-(26). 


\section{Solution I}

In order to integrate (27) let us assume

$$
\left.\begin{array}{l}
C_{2}=\alpha C_{3} \\
C_{1} \equiv \text { Const } \\
\alpha=\text { constant }
\end{array}\right\}
$$

then replacing (28) into (27) we get:

$$
C_{3} \ddot{C}_{3}-\frac{3}{2} \dot{C}_{3}^{2}-\frac{2\left(C_{1}-\alpha\right) r_{\Sigma}}{\alpha r_{\Sigma}^{2}+1} C_{3} \dot{C}_{3}+\frac{2\left(C_{1} r_{\Sigma}^{2}+1\right)}{\left(\alpha r_{\Sigma}^{2}+1\right)^{2}}\left[\alpha\left(\alpha-2 C_{1}\right) r_{\Sigma}^{2}+\left(C_{1}-2 \alpha\right)\right] C_{3}^{2}=0
$$

which, in terms of the new variable $C_{3}(t)=u^{-2}(t)$, becomes:

$$
\ddot{u}-\frac{2\left(C_{1}-\alpha\right) r_{\Sigma}}{\alpha r_{\Sigma}^{2}+1} \dot{u}-\frac{\left(C_{1} r_{\Sigma}^{2}+1\right)}{\left(\alpha r_{\Sigma}^{2}+1\right)^{2}}\left[\alpha\left(\alpha-2 C_{1}\right) r_{\Sigma}^{2}+\left(C_{1}-2 \alpha\right)\right] u=0
$$

The equation above allows the following three solutions:

Case I $\quad\left(C_{1}-\alpha\right)^{2} r_{\Sigma}^{2}+\left(C_{1} r_{\Sigma}^{2}+1\right)\left[\alpha\left(\alpha-2 C_{1}\right) r_{\Sigma}^{2}+\left(C_{1}-2 \alpha\right)\right]>0$

$$
\left.\begin{array}{rl}
C_{3}(t) & =\left[\beta_{1} e^{\left(\frac{\left(C_{1}-\alpha\right) r_{\Sigma}+\sqrt{\left(C_{1}-\alpha\right)^{2} r_{\Sigma}^{2}+\left(C_{1} r_{\Sigma}^{2}+1\right)\left[\alpha\left(\alpha-2 C_{1}\right) r_{\Sigma}^{2}+\left(C_{1}-2 \alpha\right)\right]}}{\alpha r_{\Sigma}^{2}+1}\right) t} t\right. \\
& \left.+\beta_{2} e^{\left(\frac{\left(C_{1}-\alpha\right) r_{\Sigma}-\sqrt{\left(C_{1}-\alpha\right)^{2} r_{\Sigma}^{2}+\left(C_{1} r_{\Sigma}^{2}+1\right)\left[\alpha\left(\alpha-2 C_{1}\right) r_{\Sigma}^{2}+\left(C_{1}-2 \alpha\right)\right]}}{\alpha r_{\Sigma}^{2}+1}\right) t}\right]^{-2}
\end{array}\right\}
$$

Case II $\quad\left(C_{1}-\alpha\right)^{2} r_{\Sigma}^{2}+\left(C_{1} r_{\Sigma}^{2}+1\right)\left[\alpha\left(\alpha-2 C_{1}\right) r_{\Sigma}^{2}+\left(C_{1}-2 \alpha\right)\right]<0$

$$
\left.\begin{array}{rl}
C_{3}(t)= & {\left[e ^ { \frac { ( C _ { 1 } - \alpha ) r _ { \Sigma } } { \alpha r _ { \Sigma } ^ { 2 } + 1 } t } \left(\beta_{1} \cos \left(\frac{\sqrt{\left(C_{1}-\alpha\right)^{2} r_{\Sigma}^{2}+\left(C_{1} r_{\Sigma}^{2}+1\right)\left[\alpha\left(\alpha-2 C_{1}\right) r_{\Sigma}^{2}+\left(C_{1}-2 \alpha\right)\right]}}{\alpha r_{\Sigma}^{2}+1}\right) t\right.\right.} \\
& \left.\left.+\beta_{2} \sin \left(\frac{\sqrt{\left(C_{1}-\alpha\right)^{2} r_{\Sigma}^{2}+\left(C_{1} r_{\Sigma}^{2}+1\right)\left[\alpha\left(\alpha-2 C_{1}\right) r_{\Sigma}^{2}+\left(C_{1}-2 \alpha\right)\right]}}{\alpha r_{\Sigma}^{2}+1}\right) t\right)\right]^{-2}
\end{array}\right\}
$$

Case III $\quad\left(C_{1}-\alpha\right)^{2} r_{\Sigma}^{2}+\left(C_{1} r_{\Sigma}^{2}+1\right)\left[\alpha\left(\alpha-2 C_{1}\right) r_{\Sigma}^{2}+\left(C_{1}-2 \alpha\right)\right]=0$

$$
C_{3}(t)=\left(\beta_{1}+\beta_{2} t\right)^{-2} e^{-\frac{2\left(C_{1}-\alpha\right) r_{\Sigma}}{\alpha r_{\Sigma}^{2}+1} t}
$$

This solution reduces to the one found in [26] when $\alpha=0$. 


\subsection{Calculation of the temperature}

It is worth calculating the temperature distribution, $T(r, t)$, for our model, through the Maxwell-Cattaneo heat transport equation. For simplicity we shall consider here the so called "truncated" version, for which it reads [18], $[21]$,

$$
\tau h^{\alpha \beta} w^{\gamma} q_{\beta ; \gamma}+q^{\alpha}=-\kappa h^{\alpha \beta}\left(T_{, \beta}+T a_{\beta}\right),
$$

where $\tau$ is the relaxation time, $\kappa$ the thermal conductivity and $h^{\alpha \beta}=g^{\alpha \beta}+$ $w^{\alpha} w^{\beta}$ the projector orthogonal to $w^{\alpha}$. Considering (2-4) then (34) becomes

$$
\tau(q B)^{\prime} B+q A B^{2}=-\kappa(T A)^{\prime} .
$$

In our case the integration of (35) gives

$$
\left.\begin{array}{rl}
T(t, r)= & {\left[f(t)+\frac{\tau}{4 \pi \kappa} \frac{\alpha r^{2}+1}{C_{1} r^{2}+1}\left(\ddot{C}_{3} C_{3}+\dot{C}_{3}^{2}\right)\right.} \\
& \left.-\frac{\dot{C}_{3}}{4 \pi \kappa} \ln \left(\frac{C_{1} r^{2}+1}{\alpha r^{2}+1}\right)\right] \frac{\alpha r^{2}+1}{C_{1} r^{2}+1}
\end{array}\right\}
$$

The integration function $f(t)$ may be easily related to the central temperature $T_{c}(t)$

$$
f(t)=T_{c}(t)-\frac{\tau}{4 \pi \kappa}\left(\ddot{C}_{3} C_{3}+\dot{C}_{3}^{2}\right)
$$

then we may write for the temperature

$$
\left.\begin{array}{rl}
T(t, r)= & {\left[T_{c}(t)+\frac{\tau}{4 \pi \kappa} \frac{\left(\alpha-C_{1}\right) r^{2}}{C_{1} r^{2}+1}\left(\ddot{C}_{3} C_{3}+\dot{C}_{3}^{2}\right)\right.} \\
& \left.-\frac{\dot{C}_{3}}{4 \pi \kappa} \ln \left(\frac{C_{1} r^{2}+1}{\alpha r^{2}+1}\right)\right] \frac{\alpha r^{2}+1}{C_{1} r^{2}+1}
\end{array}\right\}
$$

where $C_{3}$ is given by either (31), (32) or (33).

In case $C_{1}=\alpha$ our system becomes a collapsing (non-dissipative) Friedmann dust sphere, as it can be checked from (24)-(26). In this latter case the temperature, as expected, is homogeneous $\left(T(t)=T_{c}(t)\right)$. Thus, models (31)-(33) provide examples where inhomogeneity is directly related to dissipation. 


\section{Solution II}

Another solution, with an interesting physical interpretation may be found by introducing the variable $v$, defined as

$$
C_{2}(t) r_{\Sigma}^{2}+C_{3}(t)=v^{-2}(t)
$$

into equation (27), obtaining:

$$
\left.\begin{array}{l}
-2 v^{-2}\left(\frac{\ddot{v}}{v}-\frac{\dot{C}_{1} r_{\Sigma}^{2}}{C_{1} r_{\Sigma}^{2}+1} \frac{\dot{v}}{v}+\frac{2 \dot{v}}{r_{\Sigma} v}\right)-\frac{2}{r_{\Sigma}} \dot{C}_{3}\left(C_{1} r_{\Sigma}^{2}+1\right) \\
+\frac{2 v^{2}\left(C_{1} r_{\Sigma}^{2}+1\right)}{r_{\Sigma}^{2}}\left(v^{-4}-2 v^{-2} C_{3}\left(2+C_{1} r_{\Sigma}^{2}\right)+3 C_{3}^{2}\left(1+C_{1} r_{\Sigma}^{2}\right)\right)=0
\end{array}\right\}
$$

Then, defining

$$
Z(t)=C_{1} r_{\Sigma}^{2}+1
$$

equation (40) becomes:

$$
\left.\begin{array}{l}
-2 v^{-2}\left(\frac{\ddot{v}}{v}-\frac{\dot{Z}}{Z} \frac{\dot{v}}{v}+\frac{2 \dot{v}}{r_{\Sigma} v}\right)-\frac{2}{r_{\Sigma}} \dot{C}_{3} Z \\
+\frac{2 Z v^{2}}{r_{\Sigma}^{2}}\left(v^{-4}-2 v^{-2} C_{3}(1+Z)+3 Z C_{3}^{2}\right)=0
\end{array}\right\}
$$

Next, introducing the new variable $C(t)$ through $C_{3}(t)=v^{-2} C(t),(42)$ may be written as:

$$
\left.\begin{array}{l}
\frac{\ddot{v}}{u}-\frac{\dot{Z}}{Z} \frac{\dot{v}}{v}+\frac{2}{r_{\Sigma}} \frac{\dot{v}}{v}-\frac{2 Z}{r_{\Sigma}} \frac{\dot{v}}{v} C+\frac{Z}{r_{\Sigma}} \dot{C} \\
-\frac{Z}{r_{\Sigma}^{2}}\left(1-2 C(1+Z)+3 Z C^{2}\right)=0
\end{array}\right\}
$$

We shall integrate this last equation by assuming:

$$
\left.\begin{array}{l}
Z(t)=1 \\
C(t)=C \equiv \text { const }
\end{array}\right\}
$$

Observe that with this specific choice, this solution becomes a particular case of solution I with $C_{1}=$ Constant $=0$ and $\alpha=\frac{1-C}{C r_{\Sigma}^{2}}$

Then (43) becomes

$$
\ddot{v}+\frac{2}{r_{\Sigma}}(1-C) \dot{v}-\frac{1}{r_{\Sigma}^{2}}\left(1-4 C+3 C^{2}\right) v=0
$$


whose general solution is:

$$
v(t)=\left(k_{1}^{2} e^{\beta_{2} t}+k_{2}^{2} e^{-\beta_{2} t}\right) e^{\beta_{1} t}
$$

with

$$
\left.\begin{array}{l}
\beta_{1}=\frac{C-1}{r_{\Sigma}} \\
\beta_{2}=\frac{1}{r_{\Sigma}} \sqrt{2-6 C+4 C^{2}} \\
k_{1} \text { and } k_{2} \text { are integration constants }
\end{array}\right\}
$$

Then for the velocity as defined by (12) we have:

$$
U=-r \frac{\left[-2(1-C) \frac{\dot{v}}{v}-\dot{C}\right] \frac{r^{2}}{r_{\Sigma}^{2}}+\left[\dot{C}-\frac{2 \dot{v} C}{v}\right]}{(1-C) \frac{r^{2}}{r_{\Sigma}^{2}}+C}
$$

which, evaluated at the boundary surface gives

$$
U_{\Sigma}=\frac{2 r_{\Sigma}\left(k_{1}^{2}\left(\beta_{2}+\beta_{1}\right)-k_{2}^{2}\left(\beta_{2}-\beta_{1}\right) e^{-2 \beta_{2} t}\right)}{k_{1}^{2}+k_{2}^{2} e^{-2 \beta_{2} t}}
$$

And for the functions $C_{2}$ y $C_{3}$ we obtain:

$$
\left.\begin{array}{l}
C_{3}(t)=v(t)^{-2} C=\frac{C e^{-2 \beta_{1} t}}{\left(k_{1}^{2} e^{\beta_{2} t}+k_{2}^{2} e^{-\beta_{2} t}\right)^{2}} \\
C_{2}(t)=\frac{(1-C) e^{-2 \beta_{1} t}}{r_{\Sigma}^{2}\left(k_{1}^{2} e^{\beta_{2} t}+k_{2}^{2} e^{-\beta_{2} t}\right)^{2}}
\end{array}\right\}
$$

For this solution, the physical variables become, using (24-26) and (50):

$$
\begin{gathered}
\mu r_{\Sigma}^{2}=\frac{3}{2 \pi v^{4}}\left[C(1-C)+r_{\Sigma}^{2} \frac{\dot{v}^{2}}{v^{2}}\left[(1-C)\left(\frac{r}{r_{\Sigma}}\right)^{2}+C\right]^{2}\right] \\
p r_{\Sigma}^{2}=\frac{1-C}{2 \pi v^{4}}\left[\left((1-C)\left(\frac{r}{r_{\Sigma}}\right)^{2}-2 C\right)+\left((1-C)\left(\frac{r}{r_{\Sigma}}\right)^{2}+C\right)^{2}\left(\frac{2 r_{\Sigma} \dot{v}}{v}+3 C-1\right)\right] \\
q r_{\Sigma}^{2}=r \frac{(1-C)}{\pi v^{6}} \frac{\dot{v}}{v}\left[\left[(1-C)\left(\frac{r}{r_{\Sigma}}\right)^{2}+C\right]^{2}\right]
\end{gathered}
$$

which, evaluated at the boundary surface take the form: 


$$
\begin{gathered}
\mu_{\Sigma} r_{\Sigma}^{2}=\frac{3}{2 \pi v^{4}}\left[\frac{r_{\Sigma}^{2} \dot{v}^{2}}{v^{2}}+C(1-C)\right] \\
p_{\Sigma} r_{\Sigma}^{2}=\frac{(1-C)}{\pi v^{4}} \frac{r_{\Sigma} \dot{v}}{v} \\
q_{\Sigma} r_{\Sigma}^{2}=\frac{(1-C)}{\pi v^{6}} \frac{r_{\Sigma} \dot{v}}{v}
\end{gathered}
$$

The expression for the temperature for this model may be obtained easily by putting $C_{1}=$ Constant $=0$ and $\alpha=\frac{1-C}{C r_{\Sigma}^{2}}$ into (38), then one obtains

$$
\left.\begin{array}{rl}
T(t, r)= & {\left[T_{c}(t)+\frac{\tau \alpha r^{2}}{4 \pi \kappa}\left(\ddot{C}_{3} C_{3}+\dot{C}_{3}^{2}\right)\right.} \\
& \left.+\frac{\dot{C}_{3}}{4 \pi \kappa} \ln \left(\alpha r^{2}+1\right)\right]\left(\alpha r^{2}+1\right)
\end{array}\right\}
$$

where $C_{3}(t)$ is given by (50). In this case, the system becomes a collapsing (non-dissipative) Friedmann dust sphere, when $\alpha=0(C=1)$. In this latter case the temperature, as expected, is homogeneous $\left(T(t)=T_{c}(t)\right)$.

The last term on the right hand side of expression (57) exhibits the influence of dissipation on the temperature, with respect to the non-dissipative case, as calculated from the non-causal (Landau-Eckart) [32, 33] transport equation, whereas the second term describes the contribution of relaxational effects. The relevance of such effects have been brought out in recent works (see [25] and references therein). In particular it is worth noticing the increasing of the spatial inhomogeneity of temperature produced by the relaxational term, an effect which has been established before [34].

Let us now present a very simple model based on the solution above. The purpose here is not the modelling of any specifical astrophysical scenario, but rather to show the feasibility of these solutions as starting point for such modelling.

Thus, let us consider the following choice of constants and initial values:

$$
\begin{gathered}
k_{1}^{2}+k_{2}^{2}=1 \\
C=1+10^{-6}
\end{gathered}
$$

and

$$
U_{\Sigma}(0)=-2.5 \times 10^{-3}
$$


Then it follows from (47) and (49) that:

$$
\begin{gathered}
\beta_{1}=\frac{10^{-6}}{r_{\Sigma}} \\
\beta_{2}=\frac{1.4142 \times 10^{-3}\left(1+10^{-6}\right)}{r_{\Sigma}} \\
k_{1}^{2}=.0577 \\
k_{2}^{2}=.9423
\end{gathered}
$$

With these values, it follows at once from (54)-(56) that $\mu_{\Sigma}(0)>q_{\Sigma}(0)=$ $p_{\Sigma}(0)>0$. Furthermore since $\mu$ is a decreasing function of $r$, these inequalities hold for all points within the sphere. As time goes on, the velocity of the boundary surface decreases (in absolute value), eventually changing of sign for a finite time value. However physical variables remains acceptable (in this model) for values of the boundary velocity close to zero, but still negative.

Thus our model describes an initially contracting and radiating sphere, approaching the equilibrium. For later times there is a bouncing of the boundary surface, however, physical variables become unphysical for this values of $t$, and the model is restricted to the collapsing regime only.

\section{Conclusion}

We have presented some exact analytical solutions to the Einstein equations, describing spherical dissipative shear-free and conformally flat collapse. The solutions are matched to the outgoing Vaidya radiating spacetime. Besides their simplicity, the merit of the models resides in the fact that they exhibit in a very clear way the influence of relaxational effects on the temperature, and thereby on the evolution of the system.

In this respect we would like to stress the modifications in the temperature profile of the models, produced by the relaxational efects. This fact cannot be over emphasized. Indeed, different temperature profiles, are not only associated with different patterns of evolution, but also, affect the luminosity 
profile, which is the most important element of observational evidence in the study of dissipative collapse.

In the same line of arguments, it is worth noticing that the resulting temperature profile for each model, will depend on the specific theory of transport employed in its calculation. Therefore, such models might be used as test-bed for different relativistic theories of dissipation.

The specific example presented at the end of the previous section, models a dissipative collapsing configuration approaching equilibrium, with all physical variables exhibiting appropriate behaviour. This support further our believe that the presented solutions may be suitable for describing astrophysical scenarios involving dissipative collapsing objects.

Finally, it is also worth noticing that density inhomogeneities are directly related to dissipation, while the space-time remains conformally flat. In the non-dissipative limit $(q=L=0)$, all models become homogeneous dust balls matched to Schwarzschild spacetime.

This reinforces doubts ( see for example [35] and references therein) on the proposal that the Weyl tensor [36] or some functions of it [37], could provide

a gravitational arrow of time. The rationale behind this idea being that tidal forces tend to make the gravitating fluid more inhomogeneous as the evolution proceeds, thereby indicating the sense of time. However, as shown in [2], density inhomogeneity, besides Weyl tensor (and the anisotropy of pressure), also depends on dissipation. The solutions obtained here, clearly bring out that dependence.

\section{Acknowledgments.}

One of us (J.O.) gratefully acknowledges financial support from the Spanish Ministerio de Educación y Ciencia through the grant BFM2003-02121 and from Junta de Castilla y Leon through grant SA0 $10 \mathrm{C} 05$.

\section{References}

[1] L Herrera and N O Santos Phys. Rev. D 70084004 (2004).

[2] L Herrera, A Di Prisco, J Martin, J Ospino, N O Santos and O Troconis Phys. Rev. D 69084026 (2004). 
[3] A Mitra Phys. Rev. D 74024010 (2006).

[4] D Kazanas and D Schramm Sources of Gravitational Radiation, L. Smarr ed., (Cambridge University Press, Cambridge) (1979).

[5] W D Arnett Astrophys. J. 218815 (1977).

[6] D Kazanas Astrophys. J. 222 L109 (1978).

[7] J Lattimer Nucl. Phys. A478 199 (1988).

[8] I Iben, Astophys. J. 138, 1090,(1963).

[9] E Myra and A. Burrows Astrophys. J. 364, 222, (1990).

[10] C Cattaneo, Atti. Semin. Mat. Fis. Univ. Modena, 3, 3 (1948).

[11] W Israel Ann. Phys., NY 100310 (1976).

[12] W Israel and J Stewart Phys. Lett. A58 213 (1976); Ann. Phys. NY 118341 (1979).

[13] B Carter, Journées Relativistes, Ed. M. Cahen, Deveber R. and Geheniahau J., (ULB, Brussels) (1976).

[14] D Pavón, D Jou and J Casas-Vázquez, Ann. Inst. H Poicaré, A36 79 (1982).

[15] W Hiscock and L Lindblom, Ann. Phys. NY, 151466 (1983).

[16] D. Jou, J. Casas-Vázquez J. and G. Lebon, Rep. Prog. Phys., 511105 (1988).

[17] D Joseph and L Preziosi, Rev. Mod. Phys. 6141 (1989).

[18] J Triginer and D Pavón, Class. Quantum Grav. 12689 (1995).

[19] D Jou, J Casas-Vazquez and G Lebon, Extended Irreversible Thermodynamics, second edition (Springer-Verlag, Berlin, 1996).

[20] D Y Tzou, Macro to Micro Scale Heat Transfer: The Lagging Behaviour, (Taylor \& Francis, Washington, 1996). 
[21] R Maartens, astro-ph/9609119

[22] A Anile, D Pavon and V Romano, gr-qc/9810014.

[23] L Herrera and D Pavón, Physica A 307121 (2002).

[24] W B Bonnor, A K G de Oliveira and N O Santos Phys. Rep. 181269 (1989).

[25] A Di Prisco, L Herrera and M Esculpi Class. Quantum Grav. 131053 (1996); A Di Prisco ,N Falcón, L Herrera, M Esculpi and N O Santos Gen. Rel. Grav. 291391 (1997); L Herrera and J Martínez Gen. Rel. Grav. 30445 (1998); M Govender, S Maharaj and R Maartens Class.Quantum.Grav. 15323 (1998); M Govender, R Maartens and S Maharaj Month.Not.R. Astron.Soc. 310557 (1999); D Schäfer and H F Goenner Gen. Rel. Grav. 322119 (2000); M Govender and K Govinder Phys.Lett.A 28371 (2001); S Wagh, M Govender, K Govinder, S Maharaj, P Muktibodh and M Moodley Class. Quantum Grav. 182147 (2001); L Herrera Phys. Lett. A 300157 (2002); R Chan, M F A da Silva and J F Villas da Rocha Int. J. Modern Phys. D 12347 (2003); N Naidu and M Govender gr-qc/0510013; N Naidu, M Govender and K Govinder gr-qc/0509088; L Herrera, A Di Prisco and W Barreto Phys. Rev. D 73024008 (2006).

[26] S Maharaj and M Govender,Int. J. Modern. Phys. D 14667 (2005).

[27] C B Collins and J Wainwright Phys. Rev. D 271209 (1983); E N Glass J. Math. Phys. 201508 (1979); P Joshi, N Dadhich and R Maartens $g r-q c / 0109051$; P Joshi, R Goswami and N Dadhich $g r-q c / 0308012$; L Herrera and N O Santos Month. Not. R. Astron. Soc., 343, 1207 (2003).

[28] L Herrera, G Le Denmat and N O Santos Int. J. Mod. Phys. D 13583 (2004).

[29] M E Cahill and G C McVittie J. Math. Phys. 111382 (1970).

[30] C W Misner and D H Sharp Phys. Rev. 136 B571 (1964).

[31] Darmois G 1927 Mémorial des Sciences Mathématiques, GauthierVillars, Paris, Fasc. 25 
[32] C Eckart C Phys. Rev. 58919 (1940).

[33] L Landau L and E Lifshitz Fluid Mechanics (Pergamon Press, London) (1959).

[34] L Herrera and N O Santos Mon. Not. R. Astron. Soc. 287161 (1997).

[35] W B Bonnor Phys. Lett. 122A 305 (1987); S W Goode, A A Coley and J Wainwright Class. Quantum Grav. 9445 (1992); T Rothman gr-qc/9906002; F Mena and R Tavakol Class. Quantum Grav. 16435 (1999); N Pelavas and K Lake Phys. Rev. D. 62 044009, (2000).

[36] R Penrose General Relativity, An Einstein Centenary Survey Ed. S W Hawking and W Israel (Cambridge: Cambridge University Press) p. 581-638 (1979).

[37] J Wainwright Gen. Rel. Grav. 16657 (1984); S W Goode and J Wainwright Class. Quantum Grav. 299 (1985); W B Bonnor Phys. Lett. 112A 26 (1985). 